\title{
Kerr Micro-combs for Radio Frequency Photonics
}

\author{
Xingyuan Xu, ${ }^{1+}$ Mengxi Tan, ${ }^{1}$ Jiayang Wu, ${ }^{1}$ Sai T. Chu, ${ }^{3}$ Brent E. Little, ${ }^{4}$ Roberto Morandotti, ${ }^{5}$ \\ Thach Nguyen ${ }^{2}$, Arnan Mitchell, ${ }^{2}$ and David J. Moss ${ }^{1, *}$ \\ ${ }^{1}$ Optical Sciences Centre, Swinburne University of Technology, Hawthorn, VIC 3122 Australia \\ ${ }^{2}$ School of Engineering, RMIT University, Melbourne, VIC 3000, Australia \\ ${ }^{3}$ Department of Physics, City University of Hong Kong, Hong Kong, China. \\ ${ }^{4} X i$ 'an Institute of Optics and Precision Mechanics Precision Mechanics of CAS, Xi'an, China \\ ${ }^{5}$ INRS -Énergie, Matériaux et Télécommunications, Varennes, Québec, J3X 1S2, Canada. \\ ${ }^{\dagger}$ Current Address: Electro-Photonics Laboratory, Dept. of Electrical and Computer Systems Engineering, \\ Monash University, Clayton, 3800 VIC, Australia \\ *dmoss@swin.edu.au
}

\begin{abstract}
We review applications of Kerr micro-combs in RF photonic systems including fractional differentiators, Hilbert Transformers and many other functions.
\end{abstract}

Radio frequency (RF) and microwave photonics, which bring together the worlds of radiofrequency engineering and optoelectronics [1], exploit the potential of optical technologies and benefit RF systems in many respects, including high speed, broad operation bandwidth, low loss, and strong immunity to electromagnetic interference [2-4]. A diverse range of photonic approaches to RF signal generation, transmission, processing, and sensing have been proposed and widely employed in RF systems and communication networks [5-13]. Nevertheless, most RF systems are composed of discrete components, which impose certain drawbacks in terms of cost, power consumption and reliability, thus holding RF photonic systems from reaching maturity and replacing traditional RF solutions [14, 15]. Meanwhile, advances in integrated photonics [16-21], driven by the compelling economics of ever smaller footprint and lower power consumption, have created new possibilities and opportunities for RF photonics. Commercialized wafer scale fabrication of III-V, dielectrics, elemental semiconductor and nonlinear crystals have solved key challenges for the co-integration of lasers, modulators, photodetectors, and passive components, and have paved the road for integrated RF photonics to bring it closer towards commercial applications.

We review our recent work on $\mathrm{RF}$ and microwave photonic applications of integrated micro-combs, including a reconfigurable RF photonic intensity differentiator, $\mathrm{RF}$ channelizer and others. By employing an on-chip nonlinear micro-ring resonator (MRR), we generate a broadband Kerr comb based on soliton crystals, with a record low FSR of $49 \mathrm{GHz}$, generating a large number of comb lines and use it as a high-quality multi-wavelength source for a transversal differentiator. By programming and shaping the power of individual comb lines according to corresponding tap weights, reconfigurable intensity differentiators with variable differentiation orders can be achieved. Detailed analyses of the operation principle and experimental demonstrations of fractional, $1^{\text {st }}, 2^{\text {nd }}$, and $3^{\text {rd }}$ order differentiations are performed.
As one of the most powerful tools in RF photonic systems, optical frequency combs can serve as multi-wavelength sources and establish multiple RF channels, and thus can greatly increase the capacity for transmission and performance for transversal processers [22-26]. Unfortunately, traditional approaches like discrete laser arrays, mode-locked lasers, or cascaded modulators all have limitations of one form or another, such as the cost, ability to be integrated or the number of available wavelengths, and thus pose challenges for integrated RF photonic systems.

Micro-comb sources, particularly those based on novel CMOS-compatible platforms [27, 28], offer new possibilities for integrated RF photonics. In 2008-10, new platforms for nonlinear optics, including Hydex [27, 29-36] and silicon nitride [37], were introduced that exhibit negligible nonlinear absorption in the telecom band, a moderate nonlinear parameter and extremely high nonlinear figure of merit, which are ideal for micro-comb generation. Following the first report of Kerr frequency comb sources in 2007 [38], the first integrated CMOS compatible integrated optical parametric oscillators were reported in 2010 [27, 37], and since then this field has exploded. Many cutting-edge applications have been demonstrated based on CMOS-compatible micro-combs, ranging from filter-driven mode-locked lasers [39-42] to quantum physics [43-49]. Meanwhile, for RF photonics, many new applications have been investigated with the fundamental advantages of micro-combs demonstrated [50-53].

As compared with conventional intensity differentiators based on laser diode arrays, the cost, size and complexity can be greatly reduced. Our scheme enables a high degree of reconfigurability in terms of processing functions and operation bandwidth, offering a reconfigurable platform for diverse microwave photonic computing functions. By programming the waveshaper's tap coefficients, our scheme can also apply to other computing functions such as Hilbert transforms. The operation bandwidth is fundamentally limited by the Nyquist zone, which is determined by the comb spacing. In our case, the frequency spacing of the Kerr comb generated by the nonlinear MRR reaches $200 \mathrm{GHz}$, thus leading to a potential operation bandwidth of over $100 \mathrm{GHz}$. 


\section{References}

[1] J. Capmany, and D. Novak, "Microwave photonics combines two worlds," Nature Photonics, $\underline{\mathbf{1}}(6), 319-330$ (2007).

[2] A. J. Seeds, "Microwave photonics," IEEE Transactions on Microwave Theory and Techniques, $\underline{\mathbf{5 0}}$ (3), 877-887 (2002).

[3] R. C. Williamson, and R. D. Esman, "RF photonics," IEEE Journal of Lightwave Technology, 26 (9-12), 1145-1153 (2008).

[4] J. P. Yao, "Microwave Photonics," IEEE Journal of Lightwave Technology, 27 (1-4), 314-335 (2009).

[5] Q. Z. Cen, et al., "Rapidly and continuously frequency-scanning optoelectronic oscillator," Optics Express, 25 (2), 635-643 (2017)

[6] J. Dai, X. Y. Xu, J. L. Ke et al., "Self-Oscillating Triangular Pulse Generator Based on 90 degrees Photonic-Assisted Phase Shifter," IEEE Photonics Technology Letters, 29 (3), 271-274 (2017).

[7] J. Dai, et al., "Self-oscillating optical frequency comb generator based on an optoelectronic oscillator," Opt Express, $\underline{23}$ (23), 30014 (2015).

[8] K. Xu, et al., "Microwave photonics: radio-over-fiber links, systems, and applications," Photonics Research, 뜨 (4), B54-B63 (2014).

[9] X. Y. Xu, J. Dai, Y. T. Dai et al., "Broadband and wide-range feedback tuning scheme for phase-locked loop stabilization of tunable optoelectronic oscillators," Optics Letters, $\underline{40}$ (24), 5858-5861 (2015).

[10] A. X. Zhang, et al., "Stable RF delivery by lambda dispersion-induced optical tunable delay," Opt Lett, $\underline{\mathbf{3 8}}$ (14), 2419 (2013).

[11] S. Mansoori, and A. Mitchell, "RF transversal filter using an AOTF," IEEE Photonics Technology Letters, 16 (3), 879-881 (2004).

[12] H. Emami, et al., "Wideband RF photonic in-phase and quadraturephase generation," Optics letters, $\underline{33}$ (2), 98-100 (2008).

[13] S. L. Pan, and J. P. Yao, "Optical generation of polarity- and shapeswitchable ultrawideband pulses using a chirped intensity modulator and a $1^{\text {st }}$ order MZ interferometer," Optics Letters, $\underline{34}$ (9), 1312-1314 (2009).

[14] D. Marpaung, et al., "Nonlinear Integrated Microwave Photonics," J. of Lightwave Technology, $\underline{32}$ (20), 3421-3427 (2014).

[15] D. Marpaung et al., "Integrated microwave photonics," Laser \& Photonics Reviews, 7 (4), 506-538 (2013).

[16] J. Wu, et al., "Compact tunable Si photonic differential-equation solver for general linear time-invariant systems," Opt Express, 22 (21), 26254 (2014).

[17] J. Y. Wu, et al., "Nested Configuration of Silicon Microring Resonator With Multiple Coupling Regimes," IEEE Photonics Technology Letters, 25 (6), 580-583 (2013).

[18] J. Y. Wu, P. Cao, T. Pan et al., "Compact on-chip 1 x 2 wavelength selective switch based on silicon microring resonator with nested pairs of subrings," Photonics Research, $\underline{\mathbf{3}}$ (1), 9-14 (2015).

[19] J. Y. Wu, et al., "On-Chip Tunable Second-Order Differential-Equation Solver Based on a Silicon Photonic Mode-Split Microresonator," J. of Lightwave Technology, 33 (17), 3542-3549 (2015).

[20] J. Y. Wu, et al., "Passive silicon photonic devices for microwave photonic signal processing," Optics Communications, 373, 44-52 (2016).

[21] J.Wu, et al., "Micro-ring resonator quality factor enhancement via an integrated Fabry-Perot cavity”, APL Photonics, 2056103 (2017).

[22] A. M. Weiner, "Microwave Photonic Filters Based on Optical Frequency Combs," 2012 Ieee Photonics Conference (IPC), 296-297 (2012).

[23] O. F. Yilmaz, et al., "True time delays using conversion/dispersion with flat magnitude response for wideband analog RF signals," Optics Express, 20 (8), 8219-8227 (2012).

[24] R. Wu, et al., "Supercontinuum-based 10-GHz flat-topped optical frequency comb generation," Optics Express, 21 (5), 6045-6052 (2013).

[25] A. J. Metcalf, et al., "High-Power Broadly Tunable Electrooptic Frequency Comb Generator," IEEE J. Selected Topics in Quantum Electronics, 19 (6), (2013).

[26] E. Hamidi, et al.,"Tunable Programmable Microwave Photonic Filters based on an Optical Frequency Comb," IEEE Trans. on Microwave Theory, $\underline{\mathbf{5 8}}$ (11), 3269-3278 (2010).

[27] L. Razzari, et al., "CMOS-compatible integrated optical hyperparametric oscillator," Nature Photonics, 4 (1), 41-45 (2010).
[28] D. J. Moss, et al., "New CMOS-compatible platforms based on silicon nitride and Hydex for nonlinear optics," Nature Photonics, $\underline{7}$ (8), 597 607 (2013).

[29] M. Ferrera, et al., "Low-power CW nonlinear optics in doped silica glass integrated waveguide structures," Nat. Photonics, $\underline{2}$ (12),737-740 (2008).

[30] D. Duchesne, et al., "Efficient self-phase modulation in low loss, high index doped silica glass integrated waveguides," Optics Express, 17 (3), 1865-1870 (2009).

[31] M. Ferrera, et al., "Low power four wave mixing in an integrated, microring resonator with $\mathrm{Q}=1.2$ million," Optics Express, 17 (16), 1409814103 (2009)

[32] M.Ferrera et al., "On-Chip ultra-fast $1^{\text {st }}$ and $2^{\text {nd }}$ order CMOS compatible all-optical integration”, Optics Express 19, (23) 23153-23161 (2011).

[33] M. Ferrera, et al., "Ultra-Fast Integrated All-Optical Integrator", Nature Communications, 1 Article 29 (2010). DOI:10.1038/ncomms1028

[34] A. Pasquazi, et al., "All-optical wavelength conversion in an integrated ring resonator,” Optics Express, 18 (4), 3858-3863 (2010).

[35] A. Pasquazi, et al., "Efficient wavelength conversion and net parametric gain via FWM in a high index doped silica waveguide," Optics Express, 18 (8), 7634-7641 (2010)

[36] M. Peccianti, et al., "Subpicosecond optical pulse compression via an integrated nonlinear chirper," Optics Express, 18 (8), 7625-7633 (2010).

[37] J. S. Levy, et al., "CMOS-compatible multiple-wavelength oscillator for on-chip optical interconnects," Nature Photonics, $\underline{4}$ (1), 37-40 (2010).

[38] P. Del'Haye, et al., "Optical frequency comb generation from a monolithic microresonator," Nature 450, (7173) 1214-1217 (2007).

[39] M.Peccianti, et al., "Demonstration of an ultrafast nonlinear microcavity modelocked laser", Nature Communications, $\underline{3} 765$ (2012).

[40] M.Kues, et. al., "Passively modelocked laser with an ultra-narrow spectral width”, Nature Photonics, 11 (3) 159 (2017).

[41] A. Pasquazi, et al., "Self-locked optical parametric oscillation in a CMOS compatible microring resonator: a route to robust optical frequency comb generation on a chip," Optics Express, $\underline{21}$ (11), 13333 13341 (2013)

[42] A. Pasquazi, et al., "Stable, dual mode, high repetition rate mode-locked laser based on a microring resonator," Optics Express, 20 (24), 27355 27362 (2012).

[43] M. Kues et al., "Quantum Optical Micro-combs", Nature Photonics $\underline{13}$ (3) 170-179 (2019). DOI:10.1038/s41566-019-0363-0.

[44] C.Reimer et al., "High-dimensional one-way quantum processing implemented on d-level cluster states", Nature Physics 15 (2) 148-153 (2019). DOI: $10.1038 / \mathrm{s} 41567-018-0347-x$

[45] C. Reimer, et al., "Integrated frequency comb source of heralded single photons," Optics Express, 22 (6), 6535-6546 (2014).

[46] C.Reimer, et al., "Cross-polarized photon-pair generation and bichromatically pumped optical parametric oscillation on a chip", Nature Communications, $\underline{6}$ Article 8236 (2015).

[47] L. Caspani, et al., "Multifrequency sources of quantum correlated photon pairs on-chip: a path toward integrated Quantum Frequency Combs," Nanophotonics, $\underline{\mathbf{5}}$ (2), 351-362 (2016).

[48] C. Reimer, et al., "Generation of multiphoton entangled quantum states by means of integrated frequency combs," Science, $\mathbf{3 5 1}$ (6278), 11761180 (2016).

[49] M.Kues, et al., "On-chip generation of high-dimensional entangled quantum states and their coherent control", Nature, $\underline{\mathbf{5 4 6}}$ (7660) 622 (2017).

[50] T. G. Nguyen, et al., "Integrated frequency comb source based Hilbert transformer for wideband microwave photonic phase analysis," Optics Express, 23 (17), 22087-22097 (2015).

[51] H. Bao et al., "Laser cavity-soliton microcombs," Nature Photonics, vol. 13, no. 6, pp. 384-389, Jun. (2019).

[52] X.Xu, et al., "Microwave Photonic All-optical Differentiator based on an Integrated Frequency Comb Source", APL Photonics, 2 (9) 096104 (2017).

[53] X. X. Xue, et al., "Programmable Single-Bandpass Photonic RF Filter Based on Kerr Comb from a Microring," IEEE J. of Lightwave Technology, $\underline{\text { 32}}$ (20), 3557-3565 (2014). 